\title{
Electromagnetic tracking for treatment verification in interstitial brachytherapy
}

\author{
Prof. Christoph Bert, PhD!, Markus Kellermeier, PhD!, Prof. Kari Tanderup, PhD2,3 \\ 'Department of Radiation Oncology, Universitätsklinikum Erlangen, Friedrich-Alexander-Universität Erlangen-Nürnberg, Erlangen, Germany, \\ ${ }^{2}$ Department of Oncology. Department of Medical Physics, Aarhus University Hospital, Aarhus, Denmark, ${ }^{3}$ Institute of Clinical Medicine, \\ Aarhus University, Aarhus, Denmark
}

\begin{abstract}
Electromagnetic tracking (EMT) is used in several medical fields to determine the position and orientation of dedicated sensors, e.g., attached to surgical tools. Recently, EMT has been introduced to brachytherapy for implant reconstruction and error detection. The manuscript briefly summarizes the main issues of EMT and error detection in brachytherapy. The potential and complementarity of EMT as treatment verification technology will be discussed in relation to in vivo dosimetry and imaging.

J Contemp Brachytherapy 20l6; 8, 5: 448-453 DOI: $10.5114 /$ jcb.2016.63356
\end{abstract}

Key words: brachytherapy, electromagnetic tracking, quality assurance.

\section{Purpose}

Interstitial brachytherapy (IBT) is a treatment option for a variety of tumor sites including, e.g., prostate [1], head and neck [2], breast [3], and gynecology [4]. Implantation of the needles/catheters (without loss of generality, all different types of needles or catheters will be referred to as catheters in this article, unless a differentiation is required) is patient specific and requires imaging such as computed tomography (CT), magnetic resonance imaging (MRI), transrectal ultrasound (TRUS), or conventional X-rays to determine the track of each implanted catheter, the so-called implant geometry. Often, this procedure is carried out manually, i.e., by identifying the trace of each catheter in the image data as part of treatment planning. As in any treatment modality, also IBT underlies the risk of errors and uncertainties. Imaging artefacts, limited resolution of the imaging data, and close proximity/overlap of the (projected) implant can deteriorate the reconstruction result with discrepancies of up to $5.5 \mathrm{~mm}[5,6]$ or even errors in catheter identification. A number of reports have been published on brachytherapy errors by international bodies including the International Commission on Radiological Protection (ICRP), the International Atomic Energy Agency (IAEA), and the GEC-ESTRO [5,7,8]. In addition, a number of databases exist that collect specific errors or events. Among them is the collection of the Nuclear Regulatory Commission (NRC) in the United States (US) [9].

During treatment plan optimization, dwell positions and dwell times are determined based on the reconstructed implant fulfilling the clinical criteria for target volumes and organs at risk (OAR). The treatment plan is electronically transferred to the afterloader control unit. Before treatment administration, patient specific quality assurance steps such as verification of the source position for a number of the channels is carried out in addition to non-patient quality assurance checking. Treatment is applied as pulsed-dose-rate (PDR) or high-dose-rate (HDR) IBT with different fractionation schedules. In current clinical practice, a single treatment plan may be optimized and used for each treatment fraction, or the same treatment plan may be used for several fractions delivered based on the same implant.

One recently investigated option to detect errors or quantify the uncertainties in IBT is electromagnetic tracking (EMT). Since many years, EMT is used in various medical applications including EM navigated punctures [10,11], cardiac mapping [12], or external beam radiation therapy [13]. Based on the generation of a precisely defined magnetic field, such systems quantify position and orientation of small dedicated sensors in the field. Recent reviews are provided by Franz et al. [14] for a broad introduction and Zhou et al. [15] with respect to EMT application in brachytherapy.

The purpose of this manuscript is to introduce the concept of treatment verification by EMT for IBT. Due to the novelty of that application, clinical data are scarce and the content will thus enlighten phantom studies and future potential of the technique.

\section{Error and uncertainty classification}

Deviations from the intended treatment outcome can be the results of errors or uncertainties. According to the Insti- 
tute of Medicine in the US, errors are defined as "the failure of planned action to be completed as intended (i.e., error of execution) or the use of a wrong plan to achieve an aim (i.e., error of planning)" [16] while uncertainty "is a parameter that characterizes the dispersion of values that can be obtained for a particular measurement when it is performed repeatedly" [17]. In principle, errors can be prevented, while uncertainties cannot be eliminated, although their magnitude may be reduced. Errors can be due to human mistakes or machine failures. In brachytherapy, errors are mainly human-caused with typical examples being "failure to identify the patient properly", wrong specification of source positions, or specifying dwell positions in the reverse order [7]. Also, the NRC lists human errors such as wrong indexer length, catheter reconstruction errors, misidentified first dwell positions, and inverted catheter direction as the most frequent errors reported in 2005-2013 [18,19]. Other potential errors include swap of catheters during reconstruction or afterloader connection, wrong intersections during implant reconstruction, or usage of wrong transfer tubes.

Influence and extent of uncertainties were recently reviewed by Kirisits et al. [5]. Among them are dosimetric influences such as source strength or limitations in the dose calculation engine (e.g., AAPM TG-43 [20,21] based algorithms ignoring heterogeneous tissues and applicator materials), but also variations in implant geometry due to moving organs, tissue swelling, or shifts of individual catheters or the whole implant.
Among the intensively discussed options to prevent errors and to minimize uncertainties are in vivo dosimetry (IVD) and imaging, ideally performed in real-time during treatment delivery $[22,23]$. Tanderup et al. reviewed the use of IVD and projected the current developments into the near future [22]. Table 1 lists the most important errors and variations of brachytherapy along with the potential for IVD. Kertzscher et al. [23] and also Tanderup and Kertzscher [19] extended this table to include the potential of real-time imaging. The main findings of Table 2 in Ref. [23] are reproduced in Table 1. Only the combination of IVD and imaging is able to detect all listed errors since patient identification is not feasible by IVD. Imaging is the method of choice for direct determination of organ motion related changes and variations in the implant position or geometry. As reported below, the motivation of some of the current investigations of EMT application in IBT is the potential of EMT to act as a complementary technique to IVD and imaging for the detection of errors and variations in IBT.

\section{Implementation of electromagnetic tracking in brachytherapy}

Electromagnetic tracking is implemented in various technical options and by several vendors [14]. Generally, the systems consist of a field generator (FG) that houses a set of coils producing in an alternating manner at least

Table 1. Errors and variations in brachytherapy along with the potential of detectability by real-time in vivo dosimetry (IVD), real-time imaging (each according to [19,22,23]), and real-time electromagnetic tracking (EMT). The probability of error and the potential effect were reported by Kertzscher et al. [23], i.e. only the classification of detectability by real-time EMT and the needed EMT coordinate systems is original in this manuscript. The EMT coordinate systems refer to: «E», the intrinsic coordinate system of the EMT device; «F» fiducial markers visible in imaging and EMT reproducibly placed during each treatment fraction; «A» an absolute crosscalibration between EMT and imaging as, e.g., reported by Bharat et al. for transrectal ultrasound and EMT [24]. A schematic drawing of the coordinate system options is shown in Figure 2. $(\boldsymbol{})$ refers to partially applicable

\begin{tabular}{|c|c|c|c|c|c|c|}
\hline \multirow[t]{2}{*}{ Quality item } & \multicolumn{3}{|c|}{ Detectability } & \multirow{2}{*}{$\begin{array}{l}\text { EMT coordinate } \\
\text { system }\end{array}$} & \multicolumn{2}{|c|}{ Error probability and effect [23] } \\
\hline & IVD & Imaging & EMT & & Probability of error & Effect \\
\hline Source calibration & $\checkmark$ & $x$ & $x$ & - & Low & Low-high \\
\hline $\begin{array}{l}\text { Afterloader source positioning and dwell } \\
\text { time (non-patient specific) }\end{array}$ & $\checkmark$ & $x$ & $(\boldsymbol{\checkmark})$ & E & - & - \\
\hline Afterloader malfunction & $\checkmark$ & $x$ & $(\boldsymbol{\Omega})$ & E & Low & Low-high \\
\hline Patient identification & $x$ & $\checkmark$ & $(\boldsymbol{})$ & $E / F$ & Low & High \\
\hline Correct treatment plan & $\checkmark$ & $x$ & $\checkmark$ & $\mathrm{E}$ & Low & High \\
\hline $\begin{array}{l}\text { Intra- and interfraction organ/applicator } \\
\text { movement }\end{array}$ & $\checkmark$ & $\checkmark$ & $\checkmark$ & $E / F / A$ & - & - \\
\hline Applicator reconstruction & $\checkmark$ & $\checkmark$ & $\checkmark$ & E & Intermediate & Low-intermediate \\
\hline $\begin{array}{l}\text { Applicator length/source } \\
\text { indexer length }\end{array}$ & $\checkmark$ & $x$ & $\checkmark$ & $E / F$ & Intermediate & Low-high \\
\hline Source step size (patient specific) & $\checkmark$ & $x$ & $\checkmark$ & $\mathrm{E}$ & Low & High \\
\hline Interchanged guide tubes & $\checkmark$ & $x$ & $\checkmark$ & $E / F$ & Intermediate & Low-high \\
\hline Recording of dose & $\checkmark$ & $x$ & $x$ & - & - & - \\
\hline
\end{tabular}




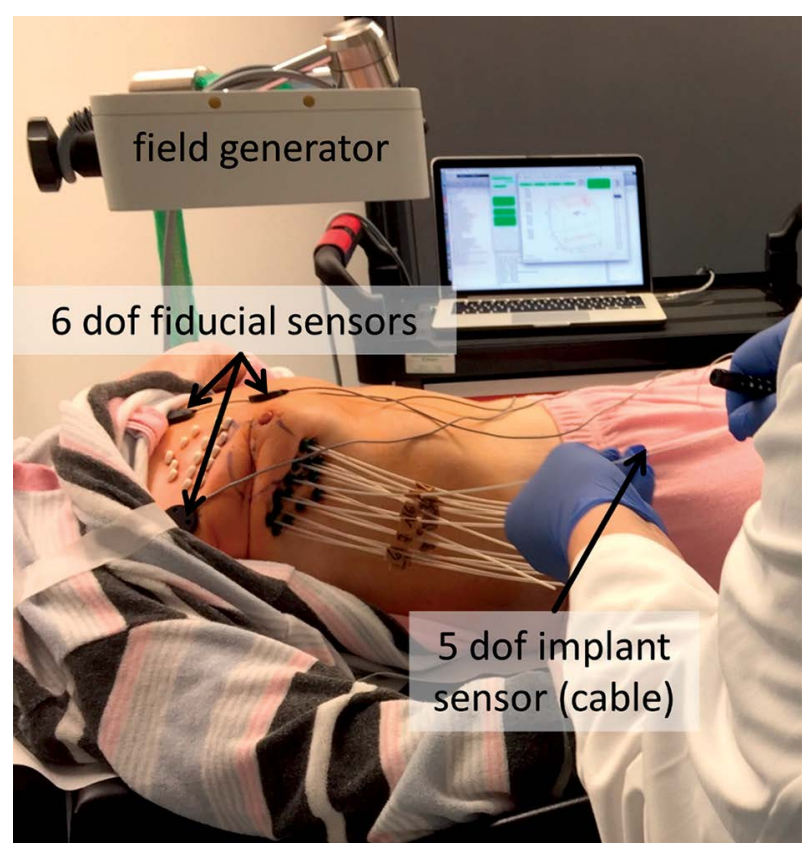

Fig. 1. Clinical use of the electromagnetic tracking system Aurora (NDI, Waterloo, Canada). The field generator spans the cubic measurement volume, in which the position and orientation of small sensors can be determined. In this example, a 5 degree of freedom (DOF) implant sensor is used to quantify the geometry of an interstitial brachytherapy breast implant. Three $6 \mathrm{DOF}$ fiducial sensors attached to the chest generate a reference coordinate system (method «F» in Table 1)

three precisely defined, inhomogeneous magnetic fields. The fields span a typical tracking volume of $500 \times 500$ $x 500 \mathrm{~mm}^{3}$ being the specification of the NDI (Waterloo, Canada) planar FG shown in Figure 1, which is used for most of the studies at Universitätsklinikum (UK) Erlangen. The magnetic field can, e.g., be measured by inductors that determine the gradient of the field at a specific point, i.e. the magnetic flux. Time-resolved measurements at $40-250 \mathrm{~Hz}$ are common for medical EMT [14] in 5 degrees of freedom (DOF) using a single 5DOF sensor (three degrees for position and two degrees for rotation), 6 DOF sensors are feasible by combining two 5DOF sensors typically requiring a larger housing.

Sensors with 5DOF are sufficiently small $(0.9-1.3 \mathrm{~mm}$ in diameter [15]) for insertion into typical catheters used for IBT. As reviewed by Zhou et al., several groups recently started to explore the potential using EMT systems from different vendors for brachytherapy [15]. The systems reach an accuracy of $<0.9 \mathrm{~mm}$ if used in environments free of disturbances such as metals that allow eddy currents disturbing the electromagnetic field. Currently, EMT is used in feasibility studies based on phantoms typically with the goal of implant definition $[24,25,26]$ or quality assurance $[27,28]$. Bharat et al. focused on prostate treatments and showed that EMT can be combined with TRUS not only "mechanically" with respect to system setup, but also regarding the measurement of coordinate systems. Electromagnetic tracking-based applicator reconstruction could therefore be easily overlaid with TRUS imaging data. Feasibility of clinical integration is currently showing at UK Erlangen where the geometry of breast implants is determined by EMT prior the irradiation in each fraction in a study approved by the institutional review board [29]. The FG is mounted on a mobile stand with a flexible arm that can quickly be positioned next to the patient in the HDR treatment room (see Figure 1). The catheters of the implant are sequentially tracked by manual insertion of a 5DOF sensor. Catheter implants of more than 30 patients have been already measured at UK Erlangen throughout their fractionated HDR-IBT treatment including measurements in the CT room.

Electromagnetic tracking-determined positions are related to the position of the typically mobile FG (coordinate system labeled «E» in Table 1 and Figure 2). In addition, EMT only "sees" sensors but does not image the patient's anatomy. A full quantitative integration of EMT with the coordinate systems used in IBT, thus requires cross-calibration of the EMT and the coordinate system of the imaging device used for specification of treatment volumes, OAR, and dwell positions. This task can be adequately achieved by identifying at least three landmarks visible in both, EMT and imaging, coordinate systems, e.g., by a reference EMT sensor attached to the stepper of the TRUS in prostate treatments in combination with probing positions with EMT-tracked needles that are visible in the TRUS data [24]. A seamless transition between the two unified coordinate systems (labeled «A» in Table 1 and Figure 2) is thus feasible, allowing, e.g., for quick and accurate implant reconstruction and overlay with segmented organs. Such co-registrations are not feasible over several days (e.g., for the treatment planning CT and EMT during each treatment fraction) unless multiple and sta-
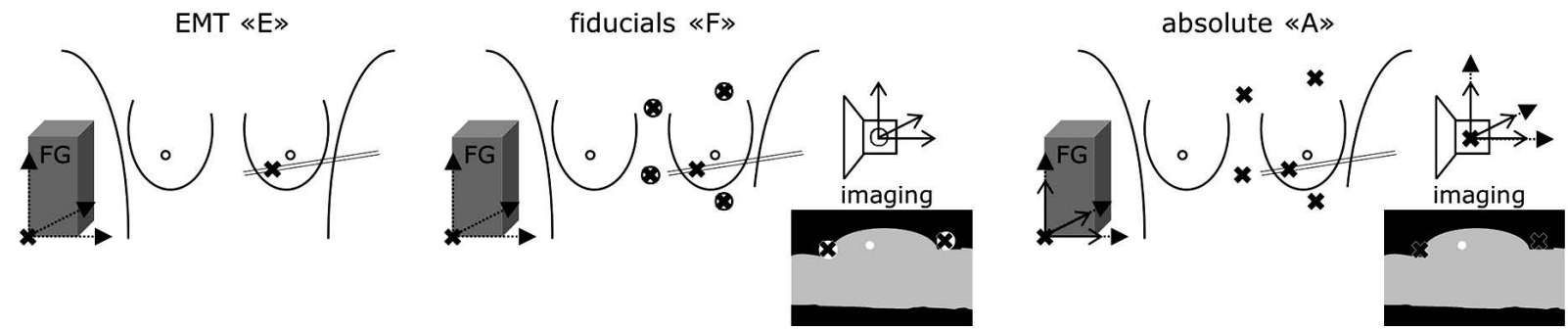

Fig. 2. Schematic of electromagnetic tracking usage in breast cancer treatments. Electromagnetic tracking can either be used standalone $(x$, method $« \mathrm{E} »)$, in combination with fiducials visible in electromagnetic tracking and an alternative imaging system $(\otimes$, method $« \mathrm{~F} »)$, or fully integrated to an imaging modality by an absolute cross-calibration (method «A») 
ble anatomical landmarks are available at the treatment site. An option would be fiducial EMT sensors placed on the skin (see Figure 1) that allow a correlation between CT imaging and each EMT measurement. The accuracy of that method (labeled $« F »$ in Table 1 and Figure 2 ) is substantially influenced by the repositioning accuracy of the fiducial sensors and uncertainties due to skin motion.

\section{Potential of electromagnetic tracking for error detection in interstitial brachytherapy}

Table 1 lists the most important errors and variations as reported by Tanderup et al. and Kertzscher et al. [19,23]. In addition to error detection by IVD and imaging as proposed by those authors, the table in the current version also lists the potential of error detection by EMT for each quality item. This judgment is not yet proven clinically, but the following paragraphs will motivate why EMT could play the indicated role.

\section{Source calibration/recording of dose}

Obviously, EMT cannot be used for dose assessment as the method determines position and orientation only.

\section{Afterloader source positioning and dwell time (non-patient specific)}

In principle, one could couple an EMT sensor with the source cable of an afterloader. Then, a fixed connection would be established between source position and EMT-determined position. Since EMT allows high frequent position determination, dwell times could also be measured. Despite high dose levels close to the EMT sensor, a reliable and accurate position reading can be expected from the EMT system $[13,30]$. As an alternative solution, the EMT sensor could be mounted to the check-cable of the afterloader. As EMT cannot detect dose, decoupling of the source from the cable could not be detected. As reported already by Tanderup et al. [22], the need for new QA options for that task is of minor importance.

\section{Afterloader malfunction}

As with the previous item, most of the potential malfunctions of the afterloader (mechanical issues, software failures, but not dosimetric properties) could be detected by EMT.

\section{Patient identification/correct treatment plan/ source step size (patient specific)}

In IBT, the catheter implant of the patient is individual regarding the implant geometry and the arrangement of dwell positions and times. Electromagnetic tracking applied just before treatment application, either by manual insertion, as performed at UK Erlangen for geometry assessments of breast implants [29], or by an automatic run of the check-cable with integrated EMT sensor of an afterloader, can determine the patient-specific implant geometry including dwell positions and dwell times. A cor- rect dosimetric outcome of the treatment plan cannot be measured, but the EMT-determined positions could be the basis for daily dose calculations. The advantage over IVD is the detection prior to application of the treatment.

\section{Intra- and interfraction organ/applicator movement}

Movement of individual catheters can be assessed by comparison to the reference implant geometry from treatment planning. Changes of the whole implant, e.g., due to swelling or other post-implant reactions, can be determined similarly. The EMT coordinate system «E» is sufficient. Relative motion of the implant site, e.g., motion of the breast/ prostate relative to important OAR could be tracked relative to EMT fiducials (method $« \mathrm{~F} »)$.

\section{Applicator reconstruction/interchanged guide tubes}

Applicator reconstruction can in principle be performed by EMT $[24,25,26]$. Without matching the coordinate systems, EMT determined implant geometries could be used as applicator/implant libraries [31] for manual overlay with the imaging data. If coordinate systems are matched (method «A») as proposed previously [24,32], the EMT determined geometry can be used directly for automatic applicator/implant placement. Potentially, EMTbased reconstruction is less prone to errors than manual reconstruction - further research is required. In case of manual reconstruction, EMT is a viable option for error detection as shown by Damato et al. with respect to mixing and interchange/swap of catheter traces [27]. Also for this quality item, EMT would spot potential errors prior to treatment delivery.

\section{Applicator length/source indexer (channel) length}

Shifts of individual catheters can be determined by EMT-only $(« E »)$ as a relative shift against the remaining catheters of the implant. Multiple individual shifts or global shifts of the whole implant can also be detected by EMT, but most likely require fiducial sensors as a reference («F»). In phantom studies, Damato et al. showed a specificity and sensitivity of each $100 \%$ for individual shifts $>2.7 \mathrm{~mm}$ [27].

\section{Conclusions}

Electromagnetic tracking is a promising option for error detection, assessment of uncertainties, and implant definition in IBT. Currently, only data from phantom-based feasibility studies are available, but these indicate that several of the typical incidences reported by IRP, IAEA, and data bases such as NRC could be detected by EMT. Therefore, clinical assessment of EMT is urgently needed as the next step, ideally well integrated to the current workflow.

A comparison to IVD and real-time imaging shows that none of these three techniques can address all of the most important error and variability items. Thus, a combination of multiple techniques will be needed. Promising combinations will depend on the clinical application, the likelihood of the errors in order to match the appro- 
priate technique, and the level of certainty desired. Several of the potential errors can be caught by EMT during the check-cable run, i.e. before dose delivery. Treatment application should then be monitored by IVD, ideally in combination with EMT [13] or an in-room imaging modality, since IVD requires precise localization of the detector. In addition, IVD and EMT integrated into the workflow can act as a trigger for follow-up imaging examinations, e.g., in case of organ deformation exceeds to be defined thresholds. Challenging for all combinations is the registration of coordinate systems needed for fusion of EMT/IVD data with the (volumetric) image set used for treatment planning. For some clinical sites, fluoroscopy, C-arm based cone-beam CT, or ultrasound may be used as an imaging modality in the treatment room, providing sufficient image quality for establishing a relation between EMT/IVD coordinates and anatomy, but not necessarily sufficient for adaptive treatments.

\section{Acknowledgments/Disclosure}

Electromagnetic tracking related research in IBT at UK Erlangen is in part funded by an unrestricted research grant from Elekta. In vivo dosimetry related research at Aarhus University Hospital is in part funded by an unrestricted research grant from Elekta.

\section{References}

1. De Bari B, Daidone A, Alongi F. Is high dose rate brachytherapy reliable and effective treatment for prostate cancer patients? A review of the literature. Crit Rev Oncol Hematol 2015; 94: 360-370.

2. Kovács G. Modern head and neck brachytherapy: from radium towards intensity modulated interventional brachytherapy. J Contemp Brachytherapy 2015; 6: 404-416.

3. Barry M, Ho A, Morrow M. The Evolving Role of Partial Breast Irradiation in Early-Stage Breast Cancer. Ann Surg Oncol 2013; 20: 2534-2540.

4. Pötter R, Haie-Meder C, Van Limbergen E et al. Recommendations from gynaecological (GYN) GEC ESTRO working group (II): Concepts and terms in 3D image-based treatment planning in cervix cancer brachytherapy - 3D dose volume parameters and aspects of 3D image-based anatomy, radiation physics, radiobiotogy. Radiother Oncol 2006; 78: 67-77.

5. Kirisits C, Rivard MJ, Baltas D et al. Review of clinical brachytherapy uncertainties: Analysis guidelines of GEC-ESTRO and the AAPM. Radiother Oncol 2014; 110: 199-212.

6. Kohr P, Siebert FA. Quality assurance of brachytherapy afterloaders using a multi-slit phantom. Phys Med Biol 2007; 52: N387-391.

7. Valentin J; International Commission on Radiation Protection. Prevention of high-dose-rate brachytherapy accidents. ICRP Publication 97. Ann ICRP 2005; 35: 1-51.

8. International Atomic Energy Agency. Safety Reports Series No. 17 - Lessons learned from accidental exposures in radiotherapy Vienna. 2000.

9. Commission, United States Nuclear Regulatory. Report to Congress on Abnormal Occurrences 2015 [updated 2.6.2015]. Available from: http://www.nrc.gov/reading-rm/doc-collections/nuregs/staff/sr0090/.

10. Wallace MJ, Gupta S, Hicks ME. Out-of-plane computed-tomography-guided biopsy using a magnetic-field-based navigation system. Cardiovasc Intervent Radiol 2006; 29: 108-113.

11. Krücker J, Xu S, Glossop N et al. Electromagnetic tracking for thermal ablation and biopsy guidance: clinical evaluation of spatial accuracy. J Vasc Interv Radiol 2007; 18: 1141-1150.

12. Linte CA, Lang P, Rettmann ME et al. Accuracy considerations in image-guided cardiac interventions: experience and lessons learned. Int J Comput Assist Radiol Surg 2012; 7: 13-25.

13. Cherpak A, Ding W, Hallil A et al. Evaluation of a novel 4D in vivo dosimetry system. Med Phys 2009; 36: 1672-1679.

14. Franz AM, Haidegger T, Birkfellner $W$ et al. Electromagnetic Tracking in Medicine-A Review of Technology, Validation, and Applications. IEEE Trans Med Imaging 2014; 33: 1702-1725.

15. Zhou J, Zamdborg L, Sebastian E. Review of advanced catheter technologies in radiation oncology brachytherapy procedures. Cancer Manag Res 2015; 7: 199-211.

16. Kohn LT, Corrigan JM, Donaldson MS (eds.). To Err is Human: Building a Safer Health System. National Academy Press, Washington 1999.

17. van Dyk J, Battista JJ, Baumann GS. Accuracy and Uncertainty Considerations in Modern Radiation Oncology. In: The Modern Technology of Radiation Oncology. van Dyk J (ed.). Vol. 3. Medical Physics Pub Corp, 2013; pp. 361-415.

18. Wilkinson DA, Kolar MD. Failure modes and effects analysis applied to high-dose-rate brachytherapy treatment planning. Brachytherapy 2013; 12: 382-386.

19. Tanderup K, Kertzscher G. Why do we need treatment verification and in vivo dosimetry 2014 [17.11.2015]. Braphyqs \& GEC-ESTRO Seminar on On-Line Treatment Verification: In Vivo Dosimetry and Computational Methods for Brachytherapy. Available from: http://www.estro.org/binaries/content/assets/estro/about/gec-estro/ivd-seminar-2014/1_ tanderup_k_seminarintroduction.pdf.

20. Nath R, Anderson LL, Luxton G et al. Dosimetry of interstitial brachytherapy sources: recommendations of the AAPM Radiation Therapy Committee Task Group No. 43. American Association of Physicists in Medicine. Med Phys 1995; 22: 209-234.

21. Rivard MJ, Coursey BM, DeWerd LA et al. Update of AAPM Task Group No. 43 Report: A revised AAPM protocol for brachytherapy dose calculations. Med Phys 2004; 31: 633-674.

22. Tanderup $K$, Beddar S, Andersen CE et al. In vivo dosimetry in brachytherapy. Med Phys 2013; 40: 070902.

23. Kertzscher G, Rosenfeld A, Beddar S et al. In vivo dosimetry: trends and prospects for brachytherapy. Br J Radiol 2014; 87: 20140206.

24. Bharat S, Kung C, Dehghan E et al. Electromagnetic tracking for catheter reconstruction in ultrasound-guided high-doserate brachytherapy of the prostate. Brachytherapy 2014; 13 : 640-650.

25. Zhou J, Sebastian E, Mangona V et al. Real-time catheter tracking for high-dose-rate prostate brachytherapy using an electromagnetic 3D-guidance device: a preliminary performance study. Med Phys 2013; 40: 021716.

26. Poulin E, Racine E, Binnekamp D et al. Fast, automatic, and accurate catheter reconstruction in HDR brachytherapy using an electromagnetic 3D tracking system. Med Phys 2015; 42: 1227.

27. Damato AL, Viswanathan AN, Don SM et al. A system to use electromagnetic tracking for the quality assurance of brachytherapy catheter digitization. Med Phys 2014; 41: 101702.

28. Kellermeier M, Herbolzheimer J, Kreppner S et al. Electromagnetic Tracking (EMT) technology for improved treatment quality assurance in interstitial brachytherapy. J Appl Clin Med Phys 2016 [In press].

29. Kellermeier M, Herbolzheimer J, Kreppner S et al. SU-FBRA-02: Electromagnetic Tracking in Brachytherapy as An Advanced Modality for Treatment Quality Assurance. Med Phys 2015; 42: 3533-3534.

30. Balter JM, Wright JN, Newell LJ et al. Accuracy of a wireless 
localization system for radiotherapy. Int J Radiat Oncol Biol Phys 2005; 61: 933-937.

31. Hellebust TP, Kirisits Ch, Berger D et al. Recommendations from Gynaecological (GYN) GEC-ESTRO Working Group: considerations and pitfalls in commissioning and applicator reconstruction in 3D image-based treatment planning of cervix cancer brachytherapy. Radiother Oncol 2010; 96: 153-160.

32. Mehrtash A, Damato A, Pernelle G et al. EM-Navigated Catheter Placement for Gynecologic Brachytherapy: An Accuracy Study. Proc SPIE Int Soc Opt Eng 2014; 9036: 90361f. 
